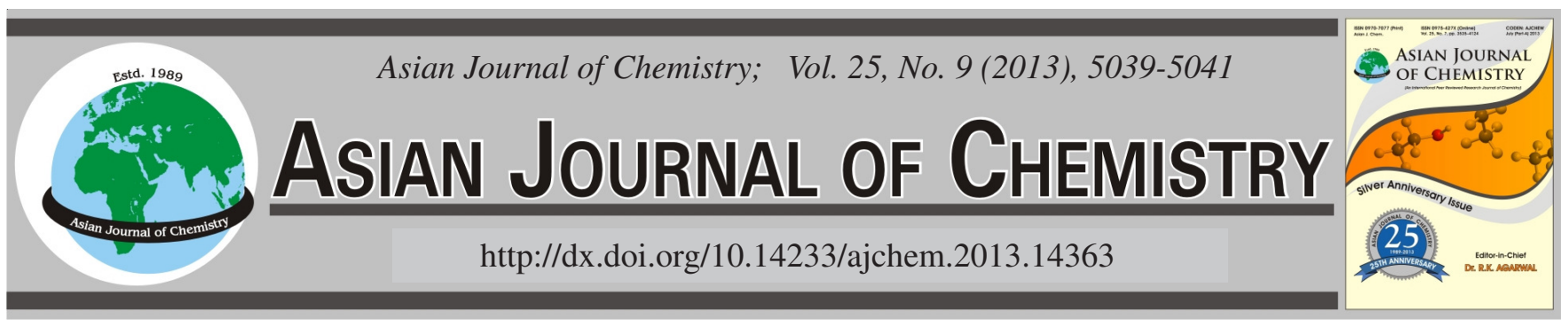

\title{
Synthesis and Crystal Structure of 6-Ethoxy-4',6'-dibromo-2,2'-[ethylenedioxybis(nitrilomethylidyne)]diphenol
}

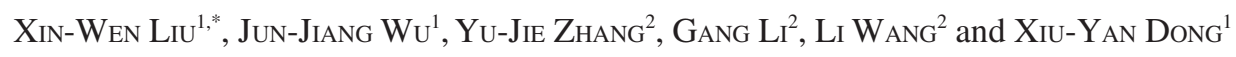

${ }^{1}$ School of Biological Science and Chemistry, Tianshui Normal University, Tianshui 741001, P.R. China

${ }^{2}$ School of Chemical and Biological Engineering, Lanzhou Jiaotong University, Lanzhou 730070, P.R. China

*Corresponding author: E-mail: liuxwts@126.com

(Received: 24 July 2012;

Accepted: 18 March 2013)

AJC-13132

The title compound, 6-ethoxy-4',6'-dibromo-2,2'-[ethylenedioxybis(nitrilomethylidyne)]diphenol with the molecular formula
$\mathrm{C}_{18} \mathrm{H}_{18} \mathrm{Br}_{2} \mathrm{~N}_{2} \mathrm{O}_{5}$, adopts an L-shaped configuration, in which the benzene units are approximately perpendicular, making a dihedral angle
of $76.39(3)^{\circ}$. Intramolecular $\mathrm{H}$-bonds are formed between the $\mathrm{OH}$ groups and the $\mathrm{N}$ atoms. In the crystal structure, each molecule links other
molecules into an infinite three-dimensional supramolecular structure by intermolecular $\mathrm{C}-\mathrm{H} \cdots \mathrm{O}$ and $\mathrm{C}-\mathrm{H} \cdots \pi$ hydrogen bond interactions.
Key Words: Asymmetric salen-type bisoxime, Synthesis, Crystal structure.

\section{INTRODUCTION}

Salen-type compounds constitute an important class of ligands which have been extensively investigated in coordination chemistry ${ }^{1}$. The development of their bisoxime analogues and complexes can provide new topologies for functional materials, in which coordination forms and functionality are significant variables ${ }^{2}$. Mainly due to their facile synthesis and easily tunable steric, electronic and catalytic properties can be used to acquire non-linear optical materials ${ }^{3}$, biological systems ${ }^{4}$, interesting magnetic properties ${ }^{5}$. They are also useful in constructing supramolecular structures ${ }^{6}$. Thus, new materials can be produced by using these compounds, which seem to be suitable candidates for further chemical modifications ${ }^{7,8}$. Herein, we report on the synthesis and crystal structure of 6-ethoxy-4',6'-dibromo-2,2'-[ethylenedioxy bis(nitrilomethylidyne)]diphenol.

\section{EXPERIMENTAL}

3,5-Dibromo-2-hydroxybenzaldehyde and 3-ethoxy-2hydroxy benzaldehyde were purchased from Alfa Aesar and used without further purification. The other reagents and solvents were analytical grade reagents from Tianjin Chemical Reagent Factory. C, H and $\mathrm{N}$ analyses were carried out with a GmbH VariuoEL V3.00 automatic elemental analyzer. IR spectra in the range 4000-400 $\mathrm{cm}^{-1}$ were recorded on a VERTEX70 FT-IR spectrophotometer using $\mathrm{KBr}$ pellets. The ${ }^{1} \mathrm{H}$ NMR spectra were recorded on a Mercury-400BB spectrometer at room temperature using $\mathrm{CDCl}_{3}$ as solvent. X-Ray single crystal structure was determined on a Bruker Smart 1000 CCD area detector. Melting points were measured by the use of a microscopic melting point apparatus made in Beijing Taike Instrument Limited Company and the thermometer was uncorrected.

General procedure: 6-Ethoxy-4',6'-dibromo-2,2'[ethylenedioxybis(nitrilomethylidyne)]diphenol was synthesized according to an analogous method reported earlier?

1,2-Bis(aminooxy)ethane was synthesized by a reported method ${ }^{10}$. Yield $75.2 \%$. Anal. calcd. (\%) for $\mathrm{C}_{2} \mathrm{H}_{8} \mathrm{~N}_{2} \mathrm{O}_{2}: \mathrm{C}$, 26.08; H, 8.76; N, 30.42. Found (\%): C, 25.99; H, 8.92; N, 30.35 .

3-Ethoxysalicylaldehyde O-(1-ethyloxyamide)oxime: A solution of 1,2-bis(aminooxy)ethane (368.2 mg, $4 \mathrm{mmol}$ ) in ethanol $(20 \mathrm{~mL})$ was added to a solution of 3-ethoxy-2hydroxybenzaldehyde (332.1 mg, $2 \mathrm{mmol}$ ) in ethanol (20 mL), the mixture was heated at $328-333 \mathrm{~K}$ for $4 \mathrm{~h}$ and concentrated under reduced pressure. The residue was purified by column chromatography $\left(\mathrm{SiO}_{2}\right.$, chloroform/ethyl acetate, 50:3) to afford $284.0 \mathrm{mg}$ crystals of the product. Yield 59.2\%. m.p. 336-337 K. Anal. calcd. (\%) for $\mathrm{C}_{11} \mathrm{H}_{16} \mathrm{~N}_{2} \mathrm{O}_{4}$ : C, 54.99; H, 6.71; N, 11.66. Found (\%): C, 54.92; H, 6.75; N, 11.69.

6-Ethoxy-4',6'-dibromo-2,2'-[ethylenedioxybis(nitrilomethylidyne)]diphenol: A solution of 3-ethoxy salicylaldehyde O-(1-ethyloxyamide)oxime (240.3 mg, $1 \mathrm{mmol}$ ) in ethanol $(20 \mathrm{~mL})$ was added to a solution of 3,5-dibromo-2hydroxybenzaldehyde (279.2 $\mathrm{mg}, 1 \mathrm{mmol})$ in ethanol $(20 \mathrm{~mL})$ and the mixture was heated at $328-333 \mathrm{~K}$ for $6 \mathrm{~h}$. After cooling to room temperature, white precipitates were collected on a suction filter to give $407.6 \mathrm{mg}$ white powder of the title 
compound. Yield: $81.2 \%$. m.p. 396-398 K. Anal. calcd. (\%) for $\mathrm{C}_{18} \mathrm{H}_{18} \mathrm{~N}_{2} \mathrm{O}_{5} \mathrm{Br}_{2}: \mathrm{C}, 43.05 ; \mathrm{H}, 3.61 ; \mathrm{N}, 5.58$. Found (\%): $\mathrm{C}$, 43.01; H, 2.93; N, 5.61.

Colourless needle-like single crystals suitable for X-ray diffraction studies were obtained after about one month by slow evaporation from a ethanol/acetone (1:1) solution of the title compound.

X-Ray structure determination: The single crystal of the title compound, with approximate dimensions of $0.32 \mathrm{~mm}$ $\times 0.10 \mathrm{~mm} \times 0.06 \mathrm{~mm}$ was placed on a Bruker Smart 1000 diffractmeter equipped with Apex CCD area detector. The diffraction data were collected using a graphite monochromated $\operatorname{MoK}_{\alpha}$ radition $(\lambda=0.71073 \AA$ ) at $298(2) \mathrm{K}$. The structure was solved by using the program SHELXS-97 and Fourier difference techniques and refined by full-matrix least-squares method on $\mathrm{F}^{2}$ using SHELXL-97. Details of the data collection and refinements of the title compound are given in Table-1. The non-hydrogen atoms were refined anisotropically. Hydrogen atoms were added theoretically. CCDC: 891145.

\section{RESULTS AND DISCUSSION}

X-Ray crystallographic analysis revealed the crystal structure of the title compound. The structure is shown in Fig. 1 and packing arrangement of the unit cell is shown in Fig. 2. Selected bond distances and angles are listed in Table-2. The structure of the title compound consists of discrete $\mathrm{C}_{18} \mathrm{H}_{18} \mathrm{~N}_{2} \mathrm{O}_{5} \mathrm{Br}_{2}$ molecule, in which all bond lengths and angles are in normal ranges. The molecule is disposed about an L-shaped configuration, in which the benzene units are approximately perpendicular, making a dihedral angle of $76.39(3)^{\circ}$. There are two weak intramolecular $\mathrm{O}-\mathrm{H} \cdots \mathrm{N}$ hydrogen bonds involving the hydroxyl groups and adjacent $\mathrm{N}$ atoms. In the crystal structure, intermolecular $\mathrm{C}-\mathrm{H} \cdots \mathrm{O}$ and $\mathrm{C}-\mathrm{H} \cdots \pi$ hydrogen bonds link the molecules, forming an infinite three-dimensional supramolecular structure $^{11-15}$.

\begin{tabular}{|c|c|}
\hline \multicolumn{2}{|c|}{$\begin{array}{c}\text { TABLE-1 } \\
\text { CRYSTAL DATA AND STRUCTURE } \\
\text { REFINEMENT FOR THE TITLE COMPOUND }\end{array}$} \\
\hline Empirical formula & $\mathrm{C}_{18} \mathrm{H}_{18} \mathrm{~N}_{2} \mathrm{O}_{5} \mathrm{Br}_{2}$ \\
\hline Formula weight & 502.16 \\
\hline Temperature & 298(2) K \\
\hline Wavelength & $0.71073 \AA$ \\
\hline Crystal system & Monoclinic \\
\hline Space group & $\mathrm{C} 2 / \mathrm{c}$ \\
\hline Cell dimensions & $\begin{array}{l}\mathrm{a}=14.760(1) \AA, \mathrm{b}=12.969(1) \AA, \\
\mathrm{c}=20.905(2), \AA \beta=100.871(1)^{\circ}\end{array}$ \\
\hline Volume & $3929.9(6) \AA^{3}$ \\
\hline $\mathrm{Z}$ & 8 \\
\hline Density (calculated) & $1.697 \mathrm{mg} / \mathrm{m}^{3}$ \\
\hline Absorption coefficient & $4.157 \mathrm{~mm}^{-1}$ \\
\hline$F_{(000)}$ & 2000 \\
\hline Index ranges & $-17 \leq \mathrm{h} \leq 17,-7 \leq \mathrm{k} \leq 15,-24 \leq 1 \leq 24$ \\
\hline Reflections collected/unique & $9261 / 3460\left[\mathrm{R}_{(\mathrm{int})}=0.0781\right]$ \\
\hline Independent reflections & 1160 \\
\hline Data/restraints/parameters & $3460 / 0 / 246$ \\
\hline Goodness of fit indicator & 1.059 \\
\hline $\mathrm{R}[\mathrm{I}>2 \sigma(\mathrm{I})]$ & $\mathrm{R}_{1}=0.0463, \mathrm{wR}_{2}=0.0851$ \\
\hline Largest diff. peak and hole & 0.809 and -0.873 e $\AA^{-3}$ \\
\hline
\end{tabular}

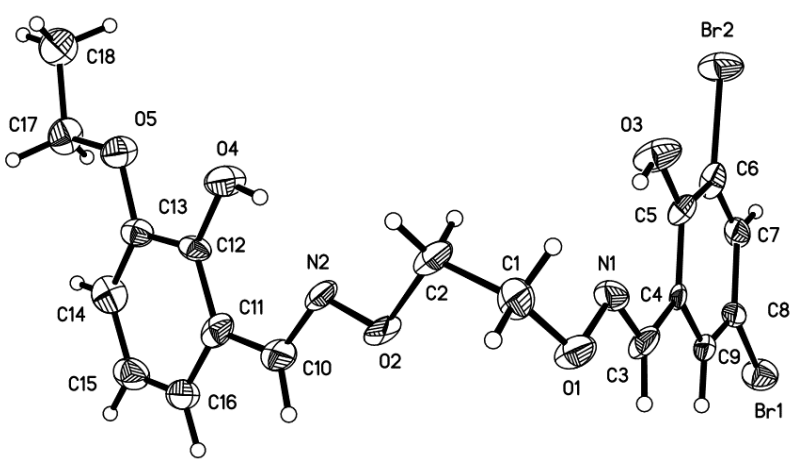

Fig. 1. Molecule structure of the title compound with atom numbering scheme. Displacement ellipsoids for non-hydrogen atoms are drawn at the $30 \%$ probability level

TABLE-2

SELECTED BOND DISTANCES $(\AA ̊)$ AND ANGLES $\left({ }^{\circ}\right)$ FOR THE TITLE COMPOUND

\begin{tabular}{cccccc}
\hline Bond & Lengths & Bond & Lengths & Bond & Lengths \\
\hline $\mathrm{Br}(1)-\mathrm{C}(8)$ & $1.838(13)$ & $\mathrm{O}(5)-\mathrm{C}(13)$ & $1.365(15)$ & $\mathrm{C}(10)-\mathrm{C}(11)$ & $1.459(18)$ \\
$\mathrm{Br}(2)-\mathrm{C}(6)$ & $1.870(14)$ & $\mathrm{O}(5)-\mathrm{C}(17)$ & $1.409(14)$ & $\mathrm{C}(11)-\mathrm{C}(12)$ & $1.358(17)$ \\
$\mathrm{N}(1)-\mathrm{C}(3)$ & $1.275(15)$ & $\mathrm{C}(1)-\mathrm{C}(2)$ & $1.512(16)$ & $\mathrm{C}(11)-\mathrm{C}(16)$ & $1.441(19)$ \\
$\mathrm{N}(1)-\mathrm{O}(1)$ & $1.401(12)$ & $\mathrm{C}(3)-\mathrm{C}(4)$ & $1.434(16)$ & $\mathrm{C}(12)-\mathrm{C}(13)$ & $1.374(17)$ \\
$\mathrm{N}(2)-\mathrm{C}(10)$ & $1.283(15)$ & $\mathrm{C}(4)-\mathrm{C}(5)$ & $1.388(16)$ & $\mathrm{C}(13)-\mathrm{C}(14)$ & $1.381(17)$ \\
$\mathrm{N}(2)-\mathrm{O}(2)$ & $1.399(12)$ & $\mathrm{C}(4)-\mathrm{C}(9)$ & $1.402(16)$ & $\mathrm{C}(14)-\mathrm{C}(15)$ & $1.381(19)$ \\
$\mathrm{O}(1)-\mathrm{C}(1)$ & $1.435(14)$ & $\mathrm{C}(5)-\mathrm{C}(6)$ & $1.406(16)$ & $\mathrm{C}(15)-\mathrm{C}(16)$ & $1.317(18)$ \\
$\mathrm{O}(2)-\mathrm{C}(2)$ & $1.421(15)$ & $\mathrm{C}(6)-\mathrm{C}(7)$ & $1.388(17)$ & $\mathrm{C}(17)-\mathrm{C}(18)$ & $1.499(18)$ \\
$\mathrm{O}(3)-\mathrm{C}(5)$ & $1.314(13)$ & $\mathrm{C}(7)-\mathrm{C}(8)$ & $1.385(16)$ & - & - \\
$\mathrm{O}(4)-\mathrm{C}(12)$ & $1.349(14)$ & $\mathrm{C}(8)-\mathrm{C}(9)$ & $1.385(16)$ & - & - \\
$\mathrm{B}(1)$ & $\mathrm{B} d$ & $\mathrm{~B}(3)-\mathrm{C}(5)-\mathrm{C}(6)$ & $119.8(12)$ & $\mathrm{C}(12)-\mathrm{C}(11)-\mathrm{C}(10)$ & $121.8(14)$ \\
$\mathrm{C}(3)-\mathrm{N}(1)-\mathrm{O}(1)$ & $110.3(11)$ & $\mathrm{C}(4)-\mathrm{C}(5)-\mathrm{C}(6)$ & $118.3(12)$ & $\mathrm{C}(16)-\mathrm{C}(11)-\mathrm{C}(10)$ & $118.4(14)$ \\
$\mathrm{C}(10)-\mathrm{N}(2)-\mathrm{O}(2)$ & $112.6(11)$ & $\mathrm{C}(7)-\mathrm{C}(6)-\mathrm{C}(5)$ & $121.2(13)$ & $\mathrm{O}(4)-\mathrm{C}(12)-\mathrm{C}(11)$ & $123.7(14)$ \\
$\mathrm{N}(1)-\mathrm{O}(1)-\mathrm{C}(1)$ & $109.7(10)$ & $\mathrm{C}(7)-\mathrm{C}(6)-\mathrm{Br}(2)$ & $120.6(9)$ & $\mathrm{O}(4)-\mathrm{C}(12)-\mathrm{C}(13)$ & $116.2(12)$ \\
$\mathrm{N}(2)-\mathrm{O}(2)-\mathrm{C}(2)$ & $109.4(9)$ & $\mathrm{C}(5)-\mathrm{C}(6)-\mathrm{Br}(2)$ & $118.2(10)$ & $\mathrm{C}(11)-\mathrm{C}(12)-\mathrm{C}(13)$ & $120.1(13)$ \\
$\mathrm{C}(13)-\mathrm{O}(5)-\mathrm{C}(17)$ & $118.0(11)$ & $\mathrm{C}(8)-\mathrm{C}(7)-\mathrm{C}(6)$ & $120.9(13)$ & $\mathrm{O}(5)-\mathrm{C}(13)-\mathrm{C}(13)$ & $116.6(12)$ \\
$\mathrm{O}(1)-\mathrm{C}(1)-\mathrm{C}(2)$ & $113.5(11)$ & $\mathrm{C}(9)-\mathrm{C}(8)-\mathrm{C}(7)$ & $117.8(12)$ & $\mathrm{O}(5)-\mathrm{C}(13)-\mathrm{C}(14)$ & $123.7(14)$ \\
$\mathrm{O}(2)-\mathrm{C}(2)-\mathrm{C}(1)$ & $108.5(10)$ & $\mathrm{C}(9)-\mathrm{C}(8)-\mathrm{Br}(1)$ & $122.7(10)$ & $\mathrm{C}(12)-\mathrm{C}(13)-\mathrm{C}(14)$ & $119.5(13)$ \\
$\mathrm{N}(1)-\mathrm{C}(3)-\mathrm{C}(4)$ & $117.3(13)$ & $\mathrm{C}(7)-\mathrm{C}(8)-\mathrm{Br}(1)$ & $119.3(11)$ & $\mathrm{C}(13)-\mathrm{C}(14)-\mathrm{C}(15)$ & $120.0(14)$ \\
$\mathrm{C}(5)-\mathrm{C}(4)-\mathrm{C}(9)$ & $119.5(12)$ & $\mathrm{C}(8)-\mathrm{C}(9)-\mathrm{C}(4)$ & $122.4(12)$ & $\mathrm{C}(16)-\mathrm{C}(15)-\mathrm{C}(14)$ & $121.5(15)$ \\
$\mathrm{C}(5)-\mathrm{C}(4)-\mathrm{C}(3)$ & $124.7(13)$ & $\mathrm{N}(2)-\mathrm{C}(10)-\mathrm{C}(11)$ & $120.0(13)$ & $\mathrm{C}(15)-\mathrm{C}(16)-\mathrm{C}(11)$ & $119.0(14)$ \\
$\mathrm{C}(9)-\mathrm{C}(4)-\mathrm{C}(3)$ & $115.8(12)$ & $\mathrm{C}(12)-\mathrm{C}(11)-\mathrm{C}(16)$ & $119.7(14)$ & $\mathrm{O}(5)-\mathrm{C}(17)-\mathrm{C}(18)$ & $106.1(12)$ \\
$\mathrm{O}(3)-\mathrm{C}(5)-\mathrm{C}(4)$ & $121.9(12)$ & & &
\end{tabular}




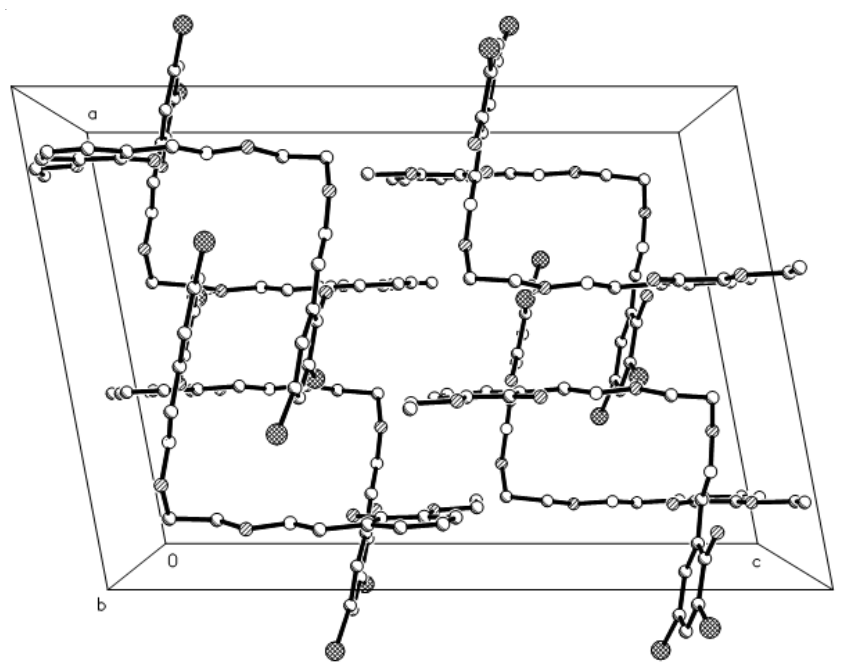

Fig. 2. Packing arrangement of the unit cell of the title compound. $\mathrm{H}$ atoms are omitted for clarity

\section{REFERENCES}

1. P.G. Cozzi, Chem. Soc. Rev., 33, 410 (2004).

2. R. Ziessel, Coord. Chem. Rev., 216, 195 (2001).
3. H. Miyasaka, N. Matsumoto, H. Okawa, N. Re, E. Gallo and C. Floriani, J. Am. Chem. Soc., 118, 981 (1996).

4. P.G. Lacroix, Eur. J. Inorg. Chem., 339 (2001).

5. T.K. Ronson, H. Adams and M.D. Ward, Inorg. Chim. Acta, 358, 1943 (2005).

6. A.K. Sharma, F. Lloret and R. Mukherjee, Inorg. Chem., 46, 5128 (2007).

7. C. Policar, F. Lambert, M. Cesario and I. Morgenstern-Badarau, Eur. J. Inorg. Chem., 12, 2201 (1999).

8. D.A. Atwood and M.J. Harvey, Chem. Rev., 101, 37 (2001).

9. W.K. Dong, Y.X. Sun, S.J. Xing, Y. Wang and X.H. Gao, Z. Naturforsch., 67b, 197 (2012).

10. W.K. Dong, Y.X. Sun, Y.P. Zhang, L. Li, X.N. He and X.L. Tang, Inorg. Chim. Acta, 362, 117 (2009).

11. W.K. Dong and J.G. Duan, J. Coord. Chem., 61, 781 (2007).

12. W.K. Dong, X.N. He, Y.X. Sun, L. Xu and Y.H. Guan, Acta Cryst., E64, 1917 (2008).

13. W.K. Dong, X.N. He, H.B. Yan, Z.W. Lv, X. Chen, C.Y. Zhao and X.L. Tang, Polyhedron, 28, 1419 (2009).

14. H.L. Wu, X.C. Huang, J.K. Yuan, F. Kou, F. Jia, B. Liu and Y. Bai, Z. Naturforsch., 66b, 1049 (2011)

15. W.K. Dong, J.G. Duan, Y.H. Guan, J.Y. Shi and C.Y. Zhao, Inorg. Chim. Acta, 362, 1129 (2009). 